\title{
A HIDDEN MARKOV MODEL FOR AN INVENTORY SYSTEM WITH PERISHABLE ITEMS
}

\author{
L. AGGOUN, L. BENKHEROUF and L. TADJ \\ King Saud University, Dept. of Statistics and Operations Research \\ P.O. Box 2455, Riyadh 11451, Saudi Arabia
}

(Received April, 1996; Revised May, 1997)

\begin{abstract}
This paper deals with a parametric multi-period integer-valued inventory model for perishable items. Each item in the stock perishes in a given period of time with some probability. Demands are assumed to be random and the probability that an item perishes is not known with certainty. Expressions for various parameter estimates of the model are established and the problem of finding an optimal replenishment schedule is formulated as an optimal stochastic control problem.
\end{abstract}

Key words: Inventory Control, Perishable Items, Markov Models, Measure Chain Techniques.

AMS subject classifications: $60 \mathrm{~K} 30,60 \mathrm{~J} 10,90 \mathrm{~B} 05$.

\section{Introduction}

This paper deals with a parametric multi-period integer valued inventory model for perishable items. Each item in the stock is assumed to perish in period $n$ with probability $\left(1-\alpha_{n}\right)$, where $0<\alpha_{n}<1$. The sequence $\left\{\alpha_{n}\right\}$ is not known with certainty but it is known to belong to one of a finite set of states of a Markov chain to be specified below. The above model is inspired from a special type of time series models called First Order Integer-Valued Autoregressive process (INAR(1)). These models were introduced independently by Al-Osh and Al-Zaid [1] and McKenzie [6] for modeling counting processes consisting of dependent variables.

Let $X$ be a nonnegative integer-valued random variable. Then for any $\alpha \in(0,1)$, define the operator o by:

$$
\alpha \circ X=\sum_{i=1}^{X} Y_{i}
$$

where $Y_{i}$ is a sequence of i.i.d. random variables, independent of $X$, such that:

$$
P\left(Y_{i}=1\right)=1-P\left(Y_{i}=0\right)=\alpha .
$$

Then the $\operatorname{INAR}(1)$ process $\left\{X_{n}: n=0, \pm 1, \pm 2, \ldots\right\}$ is given by:

$$
X_{n}=\alpha \circ X_{n-1}+V_{n}
$$

where $V_{n}$ is a sequence of uncorrelated nonnegative integer-valued random variables 
with mean $\mu_{n}$ and variance $\sigma_{n}^{2}$.

One possible application of model (1.3) is in its monitoring the number of patients in a hospital. Here the number $X_{n}$ of patients at epoch $n$ is composed of patients left from the previous epoch (each patient stays in the hospital with probability $\alpha$ or leaves with probability $(1-\alpha)$ ), and a new set $V_{n}$ of arriving patients.

Now let $X_{n}$ represent the number of items at the end of period $n$ in our inventory and consider the following extension of (1.3):

$$
X_{n}=\alpha_{n} \circ X_{n-1}-V_{n}+U_{n}
$$

with $X_{0}$ constant (integer). Here $\alpha_{n} \circ X_{n-1}=\sum_{i=1}^{n-1} Y_{i}^{n}$ as defined in (1.1) for nonnegative integer random variables $X_{n-1}$ and we allow $X_{n-1}$ to take on negative values in which case $\alpha_{n} \circ X_{n-1} \equiv 0$. Note that a negative $X_{n}$ is interpreted as shortage. $V_{n}$ is a sequence of $\mathbb{Z}^{+}$-valued independent random variables with probability distribution $\phi_{n}$ and independent of $Y_{i}^{n}$. The variable $U_{n}$ is a $\mathbb{Z}^{+}$-valued sequence representing the replenishment quantity at time $n$ (which later on will be considered as a control variable). Further we assume that the sequence $\left\{\alpha_{n}\right\}$ is a homogeneous Markov chain with finite state space $S$. For instance, $\left\{\alpha_{n}\right\}$ expresses changes in $\alpha$ caused by changes in the environment (temperature, humidity, air pressure, etc.) at different epochs $n$.

The objective of this paper is to:

(i) find the conditional probability distribution of $\left\{\alpha_{n}\right\}$ given the information accumulated about the level of inventory $\left\{X_{n}\right\}$ up to time $n$,

(ii) estimate the transition probabilities of the Markov chain $\left\{\alpha_{n}\right\}$,

(iii) formulate the optimal stochastic control problem related to (1.4).

The proposed model is an example of a hidden Markov model. Our approach shall use measure change techniques (see Elliot, Aggoun, and Moore [5]). A reference probability measure is introduced under which processes of interest are independent of each other. This greatly simplifies computations and allows derivations of interesting results.

Note that in analogy with engineering applications of hidden Markov models, we may think of $\left\{\alpha_{n}\right\}$ as a signal hidden in some noisy environment and can only be observed through noisy observations or measurements given by $\left\{X_{n}\right\}$.

Most of existing inventory models for perishable items in the literature are concerned with determining suitable ordering policies that optimize some of their performance measures. According to Nahmias [7], all perishable models fall in two main categories:

(a) fixed life perishability models,

(b) random lifetime models.

Our model is closely related to $(b)$. However, due to the complexity inherent to the analysis of models where units have a random lifetime, very little progress has been made on that front, apart from suggesting equivalent deterministic or using queueing models for their analysis. Our paper proposes a new model for perishable items which we believe is useful. For related deterministic models see Benkherouf and Mahmoud [2] and Benkherouf [3].

The paper is organized as follows: In Section 2 recursive unnormalized conditional distributions of $\left\{\alpha_{n}\right\}$ given the information accumulated about the inventory level- 
surviving items processes are derived. Section 3 contains estimates of the probability transitions of the Markov chain $\left\{\alpha_{n}\right\}$. Finally, in Section 4, an optimal control formulation for finding the replenishment schedule is proposed. The last section contains a summary and some open problems.

\section{Conditional Probability Distribution of $\left\{\alpha_{n}\right\}$}

Let $I_{1}, \ldots, I_{M}$ be a partition of the interval $(0,1)$. Also, let $s_{1}$ be any point in $I_{1}, s_{2}$ be any point in $I_{2}, \ldots, s_{M}$ be any point in $I_{M}$, and $S=\left\{s_{1}, \ldots, s_{M}\right\}$.

Suppose that the sequence $\left\{\alpha_{n}\right\}$ is a homogeneous Markov chain with state space $S$ describing the evolution of the parameter $\alpha_{n}$ of the inventory model.

All random variables are initially defined on a probability space $(\Omega, \mathcal{F}, P)$. For $1 \leq i, j \leq M$, write

and

$$
\begin{gathered}
p_{i j}=P\left(\alpha_{n} \in I_{i} \mid \alpha_{n-1} \in I_{j}\right) \\
=P\left(\alpha_{n}=s_{i} \mid \alpha_{n-1}=s_{j}\right)
\end{gathered}
$$

$$
P=\left(p_{i j}\right), \sum_{i=1}^{M} p_{i j}=1 .
$$

Recall that $X_{n}$ represents the inventory level at time $n$. Now let

and

$$
\begin{gathered}
\mathcal{A}_{n}=\sigma\left\{\alpha_{k}, k \leq n\right\} \\
\mathcal{F}_{n}=\sigma\left\{X_{k}, Y_{i}^{k}, k \leq n, i \geq 1\right\},
\end{gathered}
$$

$$
G_{n}=\sigma\left\{X_{k}, Y_{i}^{k}, \alpha_{k}, k \leq n, i \geq 1\right\},
$$

be the complete filtrations generated by the parameter process $\left\{\alpha_{n}\right\}$, the inventory level-surviving items processes $\left\{X_{n}, Y_{i}^{n}\right\}$, and the inventory level-surviving items and the parameter processes respectively.

Note that $\mathcal{A}_{n}$ and $\mathcal{F}_{n} \subset G_{n}$ for $n=1, \ldots$ Following the techniques of Elliot, Aggoun, and Moore [5], we introduce a new probability measure $\bar{P}$ under which $\left\{\alpha_{n}\right\}$ is a sequence of i.i.d. random variables uniformly distributed on the set $S=$ $\left\{s_{1}, \ldots, s_{M}\right\}$ and $\left\{X_{n}\right\}$ is a sequence of independent $\mathbb{Z}$-valued random variables with probability distributions $\phi_{n}$ independent of $\left\{\alpha_{n}\right\}$. For this, define the factors:

$$
\begin{gathered}
\lambda_{0}=1, \\
\lambda_{n}=\prod_{i=1}^{M}\left(M a_{n}^{i}\right)^{-I\left(\alpha_{n}=S_{i}\right)}\left\{\frac{\phi_{n}\left(X_{n}-\alpha_{n} \circ X_{n-1}-U_{n}\right)}{\phi_{n}\left(X_{n}\right)}\right\}^{-1},
\end{gathered}
$$

where $I\left(\alpha_{n}=s_{i}\right)$ is the indicator function of the event $\left(\alpha_{n}=s_{i}\right)$ and

$$
a_{n}^{i}=\sum_{j=1}^{M} I\left(\alpha_{n-1}=s_{i}\right) p_{i j} .
$$

Remark 1: Note that $\left\{a_{n}^{i}\right\}$ is $\mathcal{A}_{n-1}$ adapted and $E\left[I\left(\alpha_{n}=s_{i}\right) \mid \mathcal{A}_{n-1}\right]=a_{n}^{i}$, so that $I\left(\alpha_{n}=s_{i}\right)-a_{n}^{i}:=\Delta_{n}^{i}$ is an $\mathcal{A}_{n}$-martingale increment.

Now define

$$
\Lambda_{n}=\prod_{k=0}^{n} \lambda_{k}
$$


Then $\left\{\Lambda_{n}\right\}$ is a $G_{n}$-martingale such that $E\left[\Lambda_{n}\right]=1$. Here $E$ denotes the expectation under $P$. We can define a new probability measure $\bar{P}$ on $\left(\Omega, \underset{n}{\stackrel{\infty}{\vee}} G_{n}\right)$ by setting

$$
\left.\frac{d \bar{P}}{d P}\right|_{G_{n}}=\Lambda_{n}
$$

The existence of $\bar{P}$ on $\left(\Omega, \underset{n}{\stackrel{\infty}{V}} G_{n}\right)$ is due to Kolmogorov's extension theorem (see, e.g., Chung [4]). Then under $\bar{P},\left\{X_{n}\right\}$ is a sequence of independent random variables with probability distributions $\phi_{n}$ and $\left\{\alpha_{n}\right\}$ is a sequence of i.i.d. random variables uniformly distributed over $S$.

Write

$$
p_{n}\left(s_{i}\right):=P\left(\alpha_{n}=s_{i} \mid \mathscr{F}_{n}\right)=E\left\{I\left(\alpha_{n}=s_{i}\right) \mid \mathscr{F}_{n}\right\}
$$

where $\mathscr{F}_{n}$ is given by $(2.2)$, and

$$
q_{n}\left(s_{i}\right):=\bar{E}\left\{I\left(\alpha_{n}=s_{i}\right) \Lambda_{n}^{-1} \mid \mathscr{F}_{n}\right\}
$$

Here $\bar{E}$ denotes the expectation under $\bar{P}$ and $\Lambda_{n}$ is given by (2.6).

Theorem 1: For $n \geq 1$, we have

and

$$
p_{n}\left(s_{i}\right)=\frac{q_{n}\left(s_{i}\right)}{\sum_{k=1}^{M} q_{n}\left(s_{k}\right)}
$$

$$
q_{n}\left(s_{i}\right)=\frac{\phi_{n}\left(X_{n}-s_{i} \circ X_{n-1}-U_{n}\right)}{\phi_{n}\left(x_{n}\right)} \sum_{j=1}^{M} p_{i j} q_{n-1}\left(s_{j}\right) .
$$

Proof: Recall that $\left.\frac{d \bar{P}}{d P}\right|_{G_{n}}=\Lambda_{n}$ is equivalent to $\left.\frac{d P}{d \bar{P}}\right|_{G_{n}}=\Lambda_{n}^{-1}$.

Now using a version of Bayes theorem (see Elliot, Aggoun, and Moore [5]), we can write:

$$
\begin{aligned}
p_{n}\left(s_{i}\right)=E\left\{I\left(\alpha_{n}=s_{i}\right) \mid \mathcal{F}_{n}\right\}=\frac{\bar{E}\left\{I\left(\alpha_{n}=s_{i}\right) \Lambda_{n}^{-1} \mid \mathscr{F}_{n}\right\}}{\bar{E}\left[\Lambda_{n}^{-1} \mid \mathcal{F}_{n}\right\}} \\
=\frac{q_{n}\left(s_{i}\right)}{\sum_{k=1}^{M} q_{n}\left(s_{k}\right)} .
\end{aligned}
$$

Working with $\bar{P}$ and using (2.4), (2.5), (2.6), and (2.9), it is easily seen that

$$
q_{n}\left({ }_{i}\right)=\frac{\phi_{n}\left(X_{n}-s_{i} \circ X_{n-1}-U_{n}\right)}{\phi_{n}\left(X_{n}\right)} \sum_{j=1}^{M} p_{i j} q_{n-1}\left(s_{j}\right)
$$

as required.

It is worth noting at this stage that if model (1.4) is replaced by

then (2.11) becomes:

$$
X_{n}=\alpha_{n-1} \circ X_{n-1}-V_{n}+U_{n},
$$

$$
q_{n}\left(s_{i}\right)=\frac{1}{\phi_{n}\left(X_{n}\right)} \sum_{j=1}^{M} \phi_{n}\left(X_{n}-s_{j} \circ X_{n-1}-U_{n}\right) p_{i j} q_{n-1}\left(s_{j}\right) \text {. }
$$

Now, if $\alpha_{0}$ is fixed, then using (2.11) the first updated conditional distribution of $\alpha_{1}$ can be obtained using the relation 


$$
q_{1}\left(s_{i}\right)=\frac{\phi_{1}\left(X_{1}-s_{i} \circ X_{0}-U_{1}\right)}{\phi_{1}\left(X_{1}\right)} p_{i, \alpha_{0}}
$$

with

$$
P\left(\alpha_{1}=s_{i} \mid \mathcal{F}_{1}\right)=p_{1}\left(s_{i}\right)=\frac{q_{1}\left(s_{i}\right)}{\sum_{k=1}^{M} q_{1}\left(s_{k}\right)}=\frac{\phi_{1}\left(X_{1}-s_{i} \circ X_{0}-U_{1}\right) p_{i, \alpha_{0}}}{\sum_{k=1}^{M} \phi_{1}\left(X_{1}-s_{k} \circ X_{0}-U_{1}\right) p_{k, \alpha_{0}}}
$$

Further updates follow by using (2.11).

\section{Estimation of the Transition Probabilities $p_{i j}$}

In Section 2, the probabilities $p_{i j}$ were assumed to be known. However, in practice we seldom know $p_{i j}$ exactly and consequently, estimates of the transition probabilities would be reasonable requirements. These estimates are calculated each time new information regarding the behavior of the system becomes available. In this model, the state space $S=\left\{s_{1}, \ldots, s_{M}\right\}$ is fixed a priori. This is done by partitioning the open unit interval into $M$ subintervals $I_{1}, \ldots, I_{M}$ according to some prior information about the behavior of $\alpha$. When, at time $n$, the new information is made available through $\mathcal{F}_{n}$-measurable estimates $\left\{\widehat{p}_{i j}(n)\right\}$, a new partition of $I$ can replace the initial one updating the state space of $\left\{\alpha_{n}\right\}$.

To replace, at time $n$, the parameter $\left\{p_{i j}(n)\right\}$ by $\left\{\widehat{p}_{i j}(n)\right\}$ in the Markov chain $\left\{\alpha_{n}\right\}$, define

$$
\widehat{\Lambda}_{n}=\prod_{k=1}^{n} \prod_{i=1}^{M} \prod_{j=1}^{M}\left(\frac{\widehat{p}_{i j}(n)}{p_{i j}}\right)^{I\left(\alpha_{k} \in I_{i}\right) I\left(\alpha_{k-1} \in I_{j}\right)}
$$

and set

$$
\left.\frac{d \widehat{P}}{d P}\right|_{G_{n}}=\widehat{\Lambda}_{n}
$$

Again, the existence of $\widehat{P}$ is due to Kolmogorov's extension theorem.

It is easy to see that under $\widehat{P}$, the Markov chain $\left\{\alpha_{n}\right\}$ has transition probabilities given by $\left\{\widehat{p}_{i j}(n)\right\}$.

Theorem 2: The new estimates $\left\{\widehat{p}_{i j}(n)\right\}$ at time $n$ of the probability transitions given the observation of the inventory level from time 0 to time $n$ are given by:

where

$$
\widehat{p}_{i j}(n)=\frac{\gamma_{n}\left(N_{n}^{j i}\right)}{\sum_{l=1}^{M} \gamma_{n}\left(N_{n}^{j l}\right)}
$$

$$
\gamma_{n}(X)=\bar{E}\left\{\Lambda_{n}^{-1} X \mid \sigma_{n}\right\}
$$

and $N_{n}^{j i}$ counts the number of transitions from state $j$ to state $i$ up to time $n$.

Proof: Note that from (3.1),

$$
\begin{gathered}
\log \widehat{\Lambda}_{n}=\sum_{k=1}^{n} \sum_{i=1}^{M} \sum_{j=1}^{M} I\left(\alpha_{k} \in I_{i}\right) I\left(\alpha_{k-1} \in I_{j}\right)\left\{\log \widehat{p}_{i j}(n)-\log p_{i j}\right\} \\
=\sum_{i=1}^{M} \sum_{j=1}^{M} N_{n}^{j i} \log \widehat{p}_{i j}(n)+R\left(p_{i j}\right)
\end{gathered}
$$


where $R\left(p_{i j}\right)$ does not contain $\widehat{p}_{i j}(n)$ terms.

Conditioning on $\mathcal{F}_{n}$,

$$
E\left(\log \widehat{\Lambda}_{n} \mid \mathcal{F}_{n}\right)=\sum_{i=1}^{M} \sum_{j=1}^{M} E\left(N_{n}^{j i} \mid \mathcal{F}_{n}\right) \log \widehat{p}_{i j}(n)+E\left\{R\left(p_{i j}\right) \mid \mathcal{F}_{n}\right\}
$$

We require that

$$
\sum_{i=1}^{M} \widehat{p}_{i j}(n)=1
$$

We wish to choose $\left\{\widehat{p}_{i j}(n)\right\}$ that maximizes (3.4) subject to (3.5). Consider the auxiliary problem:

$$
L\left(\widehat{p}_{i j}(n), \lambda\right)=E\left(\log \widehat{\Lambda}_{n} \mid \digamma_{n}\right)+\lambda\left(\sum_{i=1}^{M} \widehat{p}_{i j}(n)-1\right) .
$$

Maximizing $L\left(\widehat{p}_{i j}(n), \lambda\right)$ gives

$$
\begin{gathered}
\frac{E\left(N_{n}^{j i} \mid \mathscr{F}_{n}\right)}{\widehat{p}_{i j}(n)}+\lambda=0 \\
\sum_{i=1}^{M} \widehat{p}_{i j}(n)=1
\end{gathered}
$$

Hence,

$$
\widehat{p}_{i j}(n)=\frac{E\left(N_{n}^{j i} \mid \mathcal{F}_{n}\right)}{\sum_{i=1}^{M} E\left(N_{n}^{j i} \mid \mathcal{F}_{n}\right)}=\frac{\bar{E}\left(N_{n}^{j i} \Lambda_{n}^{-1} \mid \mathcal{F}_{n}\right)}{\sum_{i=1}^{M} \bar{E}\left(N_{n}^{j i} \Lambda_{n}^{-1} \mid \mathcal{F}_{n}\right)}=\frac{\gamma_{n}\left(N_{n}^{j i}\right)}{\sum_{i=1}^{M} \gamma_{n}\left(N_{n}^{j i}\right)}
$$

Next, we derive a recursive equation for the unnormalized conditional probability $\gamma_{n}\left(N_{n}^{j i}\right)$. However, in order to obtain a recursion for $\gamma_{n}\left(N_{n}^{j i}\right)$, we derive first recursive equations for the process $\left\{I\left(\alpha_{n}=s_{l}\right) N_{n}^{j i}\right\}$ as a necessary intermediate step:

Note that

$$
\bar{E}\left\{I\left(\alpha_{n}=s_{l}\right) N_{n}^{j i} \Lambda_{n}^{-1} \mid \mathscr{F}_{n}\right):=\tilde{\gamma}_{n}\left\{I\left(\alpha_{n}=s_{l}\right) N_{n}^{j i}\right\} .
$$

$$
\gamma_{n}\left(N_{n}^{j i}\right)=\sum_{l=1}^{M} \tilde{\gamma}_{n}\left\{I\left(\alpha_{n}=s_{l}\right) N_{n}^{j i}\right\}
$$

Theorem 3: For $n \geq 1$,

$$
\tilde{\gamma}_{n}\left\{I\left(\alpha_{n}=s_{l}\right) N_{n}^{j i}\right\}
$$

$$
=\frac{\phi_{n}\left(X_{n}-\alpha_{n} \circ X_{n-1}-U_{n}\right)}{\phi_{n}\left(X_{n}\right)}\left[\sum_{m=1}^{M} \tilde{\gamma}_{n}\left\{I\left(\alpha_{n-1}=s_{m}\right) N_{n-1}^{j i}\right\} p_{l m}+q_{n-1}^{(j)} p_{l j} p_{i j}\right] \text {, }
$$

where $q_{n}$ is given recursively by (2.11).

Proof: The proof follows if we note that

$$
N_{n}^{j i}=N_{n-1}^{j i}+I\left(\alpha_{n-1}=s_{j}\right) I\left(\alpha_{n}=s_{i}\right),
$$

and use Remark 1, Section 2.

The revised parameters $\left\{\widehat{p}_{i j}(n)\right\}$ give new probability measures for the model. The quantities $\gamma_{n}\left(N_{n}^{j i}\right)$ can then be reestimated using the new probabilities. 


\section{Optimal Control}

In this section we formulate an optimal control problem for model (1.4). However, $\left\{\alpha_{n}\right\}$ is not fully observed. Using results of Section 2 we transform the problem into a fully observed one. Dynamic programming results and minimum principle are obtained in terms of separated controls (i.e., controls which depend on the Markov chain only through $q_{n}$ ).

Recall from (1.4) that our inventory model has the form:

$$
X_{n}=\alpha_{n} \circ X_{n-1}-V_{n}+U_{n}
$$

Assume that at each time $n, 0 \leq n \leq T$, the control $U_{n}$ takes on values in some finite subset $\mathcal{U}$ of $\mathbb{Z}^{+}$and that $U_{n}$ is $\mathcal{F}_{n}$-measurable, where $\mathscr{F}_{n}$ is given by (2.2). Also assume the existence of bounded measurable functions $c_{n}, d_{n}$, and $f_{n}$.

For each admissible control $U=\left(U_{0}, \ldots, U_{n}\right) \in \mathcal{U}$, we define the associated expected costs as:

$$
\begin{gathered}
J\left(X_{0}, U\right)=E\left[\sum_{n=0}^{T}\left\{c_{n}\left(X_{n}\right)+d_{n}\left(U_{n}\right)+f_{n}\left(\alpha_{n}\right)\right\}\right] \\
=\bar{E}\left[\Lambda_{T}^{-1} \sum_{n=0}^{T}\left\{c_{n}\left(X_{n}\right)+d_{n}\left(U_{n}\right)+f_{n}\left(\alpha_{n}\right)\right\}\right] \\
=\bar{E}\left[\sum_{n=0}^{T} \bar{E}\left(\Lambda_{n}^{-1}\left\{c_{n}\left(X_{n}\right)+d_{n}\left(U_{n}\right)+f_{n}\left(\alpha_{n}\right)\right\} \mid \mathcal{F}_{n}\right)\right] \\
=\bar{E}\left[\sum_{n=0}^{T}\left(\left\{c_{n}\left(X_{n}\right)+d_{n}\left(U_{n}\right)\right\} \bar{E}\left[\Lambda_{n}^{-1} \mid \mathcal{F}_{n}\right]+\sum_{k=1}^{M} f_{n}\left(s_{k}\right) \bar{E}\left[I\left(\alpha_{n}=s_{k}\right) \Lambda_{n}^{-1} \mid \mathcal{F}_{n}\right]\right)\right] \\
=\bar{E}\left[\sum_{n=0}^{T}\left(\left\{c_{n}\left(X_{n}\right)+d_{n}\left(U_{n}\right)\right\} \sum_{l=1}^{M} q_{n}(l)+\sum_{k=1}^{M} f_{n}(k) q_{n}(k)\right)\right],
\end{gathered}
$$

where $q_{n}(\cdot)$ are given by Theorem 1 .

So, the expected cost is expressed in terms of the conditional measures $q_{n}(\cdot)$.

The value function for this control problem is as follows for $0 \leq t \leq T$ :

$$
\begin{gathered}
V_{t}(q)_{\left(U_{t}, \ldots, U_{T}\right)} \bar{E}\left[\sum_{n=t}^{T}\left(\left\{c_{n}\left(X_{n}\right)+d_{n}\left(U_{n}\right)\right\} \sum_{l=1}^{M} q_{n}(l)+\sum_{k=1}^{M} f_{n}(k) q_{n}(k)\right) \mid q_{t}=q\right] \\
\vdots=\inf _{\left(U_{t}, \ldots, U_{T}\right)} V_{t}(q, U) .
\end{gathered}
$$

The following dynamic programming result can easily be established.

Theorem 4:

$$
V_{t}(q)=\inf _{U_{t}} \bar{E}\left[\left\{c_{t}\left(X_{t}\right)+d_{t}\left(U_{t}\right)\right\} \sum_{l=1}^{M} q_{t}(l)+\sum_{k=1}^{M} f_{t}(k) q_{t}(k)+V_{t+1}\left(q_{t+1}\right) \mid q_{t}=q\right]
$$


A minimum principle has the following form.

Theorem 5: Suppose $U^{*}$ is a control such that for each positive measure $q$ on $S=\left\{s_{1}, \ldots, s_{M}\right\}, U_{t}^{*}(q)$ achieves the minimum in $(4.4)$. Then $V_{t}\left(q, U^{*}\right)=V_{t}(q)$ and $U^{*}$ is an optimal control.

\section{Summary and Open Problems}

In this paper, an integer-valued inventory model for perishable items was introduced. Various parameters estimators of the model were suggested using measure change techniques. Further an optimal control formulation was set for the model. Possible extensions of the paper include:

1. Extending model (1.4) to take into account the age of the items. Here difficulties arise regarding the choice of the issuing policies.

2. The current paper departed from the classical approach of determining suitable ordering policies. It would be of interest to investigate Economic Order Quantity models.

3. The parameter process $\left\{\alpha_{n}\right\}$ is assumed to be a Markov chain in this paper. More general processes for $\left\{\alpha_{n}\right\}$ could be taken.

\section{References}

[1] Al-Osh, M.A. and Al-Zaid, A.A., First-order integer-valued autoregressive (INAR(1)) process, J. Time Series Anal. 8 (1987), 261-275.

[2] Benkherouf, L., On an inventory model with deteriorating items and decreasing time-varying demand and shortages, European J. of Operations Research 86 (1995), 293-299.

[3] Benkherouf, L. and Mahmoud, M.G., On an inventory model for deteriorating items with increasing time-varying demand and shortages, J. Oper. Res. Soc. 47 (1996), 188-200.

[4] Chung, K.L., A Course in Probability Theory, Academic Press, New York 1974.

[5] Elliot, R.J., Aggoun, L. and Moore, J.B., Hidden Markov Models: Estimation and Control, Applications of Mathematics 29, Springer-Verlag, New York 1995.

[6] McKenzie, E., Some simple models for discrete variate time series, Water Res. Bull. 21 (1985), 645-650.

[7] Nahmias, S., Perishable inventory theory: A review, Operations Res. 30 (1982), 680-707. 


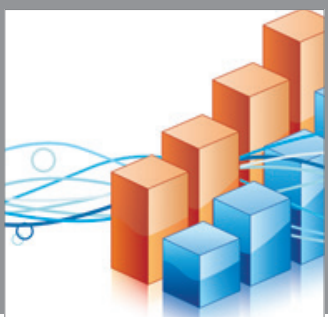

Advances in

Operations Research

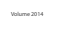

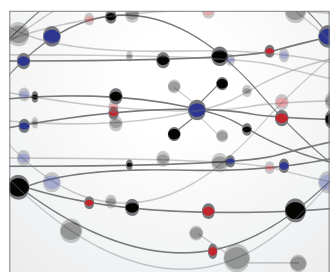

\section{The Scientific} World Journal
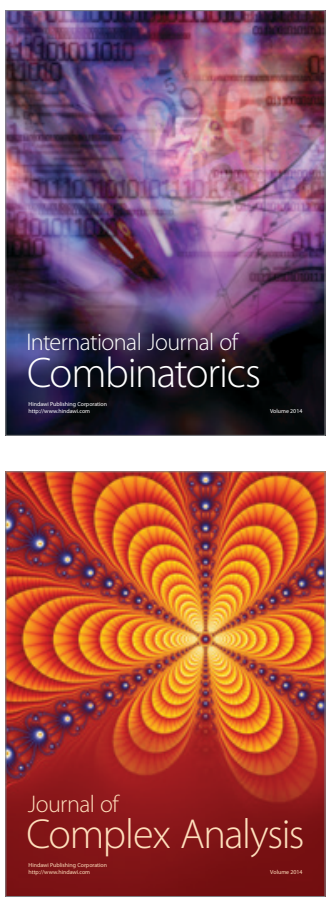

International Journal of

Mathematics and

Mathematical

Sciences
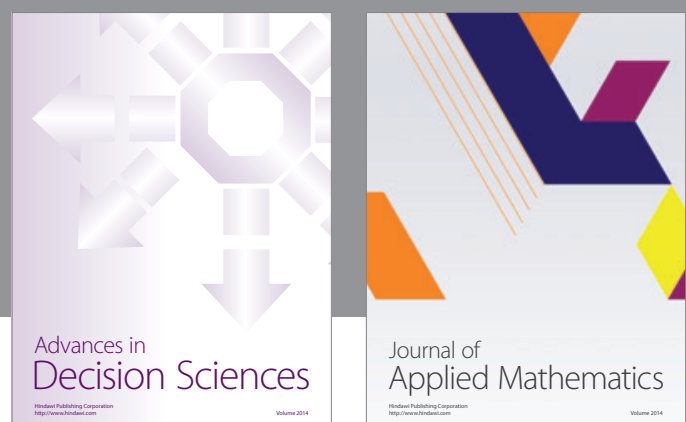

Journal of

Applied Mathematics
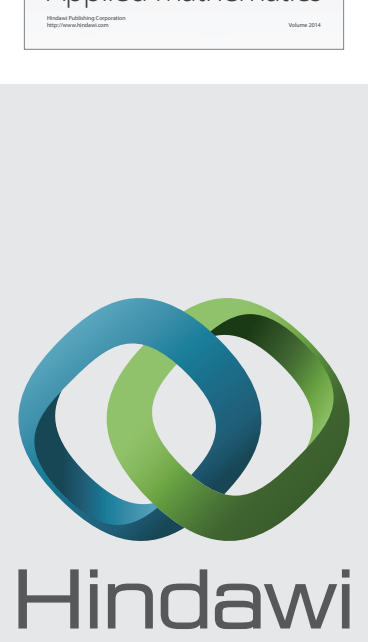

Submit your manuscripts at http://www.hindawi.com
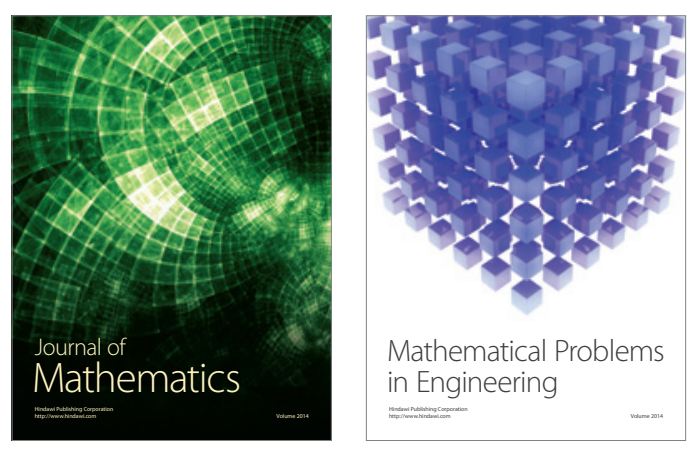

Mathematical Problems in Engineering
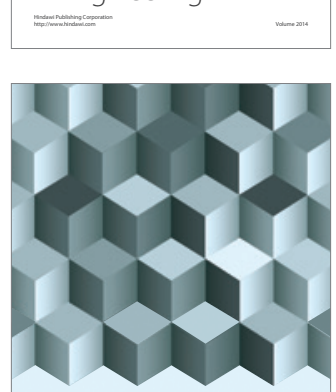

Journal of

Function Spaces
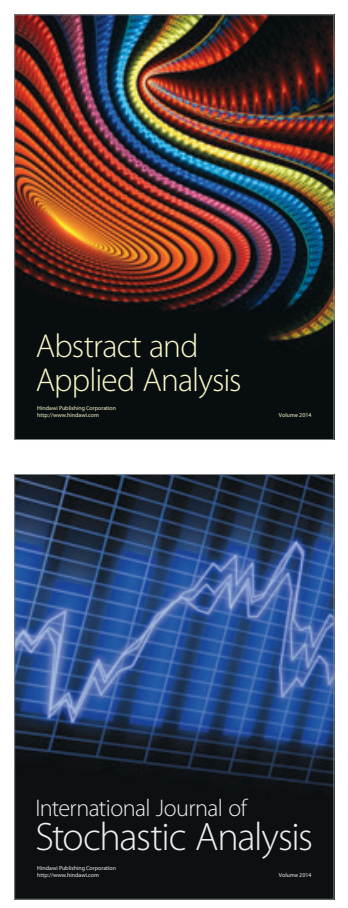

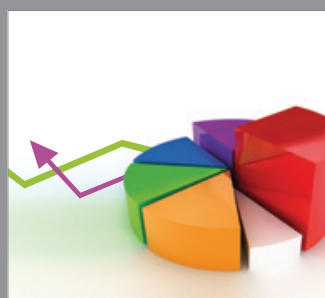

ournal of

Probability and Statistics

Promensencen
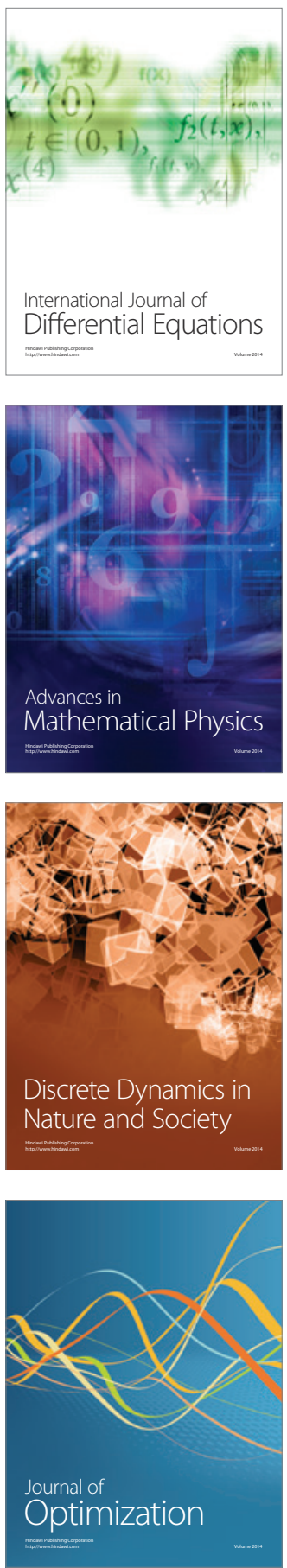